\title{
Risco de quedas, força muscular periférica e capacidade funcional em idosos hospitalizados
}

\author{
Risk of falls, peripheral muscle strength and functional capacity in \\ hospitalized elderly
}

\author{
Bruna Sutil ${ }^{1}$ \\ Andréia De Carli ${ }^{2}$ \\ Alessandra Albuquerque Donato ${ }^{3}$ \\ Cassandra Pértile Vieira ${ }^{4}$ \\ Tainara Fontana ${ }^{5}$

\section{Carla Wouters Franco Rockenbach ${ }^{6}$ \\ Lia Mara Wibelinger ${ }^{7}$} \\ Endereço para Correspondência: \\ Alessandra Albuquerque Donato \\ Universidade de Passo Fundo (UPF), BR 285 - São José \\ 99001-970 - Passo Fundo - RS [Brasil] \\ alessandra.albuquerquedonato@hotmail.com
}

\begin{abstract}
Resumo
Introdução: a longevidade vem acompanhada pelo declínio das capacidades funcionais, especialmente em idosos hospitalizados. Objetivo: avaliar o risco de queda, a força muscular periférica e a capacidade funcional em idosos hospitalizados. Métodos: participaram 100 voluntários que foram submetidos à avaliação fisioterapêutica, por meio da escala de Kendall, força de preensão palmar, teste Time Up\&Go, escala de Morse e escala de PS-ECOG. Resultados: em relação ao risco de queda, $46 \%$ dos indivíduos apresentaram risco moderado e 37\% revelaram funcionalidade restrita para atividades exaustivas. Na realização do teste de força muscular periférica, o resultado teve média geral de $4,47 \mathrm{~kg} / \mathrm{f}$, configurando força muscular moderada, para força de preensão palmar os indivíduos atingiram resultados insatisfatórios, apresentando no membro dominante média de $17,7 \mathrm{Kg} / \mathrm{f}$ e no membro contralateral média de $17,3 \mathrm{Kg} / \mathrm{f}$. Na variável "Timed Up\&Go", os indivíduos obtiveram um tempo de 12,25 segundos, no qual foram classificados como independentes. Conclusão: Os idosos hospitalizados apresentaram moderado risco de queda, capacidade funcional reduzida, embora sejam classificados como independentes.
\end{abstract}

Descritores: Longevidade. Hospitalização. Força muscular. Limitação da mobilidade. Equilíbrio postural.

\begin{abstract}
Introduction: Longevity is surrounded by declining functional abilities, especially in hospitalized elderly. Objective: Assess the risk of falling, peripheral muscle strength and functional capacity in hospitalized elderly. Methods: One hundred volunteers participated; these were submitted to physical therapy evaluation through the Kendall scale, palmar grip strength, Time Up\&Go test, Morse scale and PS-ECOG scale. Results: Regarding the risk of falls, $46 \%$ of the individuals presented moderate risk and $37 \%$ obtained restricted functionality for exhaustive activities. In the performance of the peripheral muscle strength test, the result was a general average of $4.47 \mathrm{~kg} / \mathrm{f}$, configuring moderate muscle strength. For palmar grip strength, the individuals obtained unsatisfactory results, presenting, in the dominant member, an average of $17.7 \mathrm{~kg} / \mathrm{f}$, and in the mean contralateral member of $17.3 \mathrm{~kg} / \mathrm{f}$. In the variable "Timed Up\&Go", individuals obtained a time of 12.25 seconds, in which they were classified as independent. Conclusion: Hospitalized elderly patients presented moderate risk of falls, with reduced functional capacity, although they were classified as independent.
\end{abstract}

Keywords: Longevity. Hospitalization. Muscle strength. Mobility limitation. Postural balance. 


\section{Introdução}

Com o aumento da população idosa em todos os países, a longevidade tornou-se um fenômeno mundial. Entretanto, esse processo traz consigo modificações no corpo do indivíduo, consideradas complexas e relacionadas à interação de diversos fatores, sejam genéticos, de estilo de vida, ou advindos de doenças crônicodegenerativas. No idoso, a redução da massa muscular, e a consequente diminuição da força muscular, conferem ao indivíduo certo declínio na sua funcionalidade ${ }^{1}$.

A funcionalidade é definida como capacidade em desempenhar tarefas que permitam cuidar de si mesmo e ter uma vida independente. Sua manutenção no processo de envelhecimento pode ser descrita como um indicador de saúde, pois muitas das alterações que ocorrem com o indivíduo, associadas a fatores ambientais e pessoais, predispõem o idoso a uma maior vulnerabilidade, o que pode comprometer a sua independência², visto que podem ocorrer alterações posturais, na marcha e no equilíbrio e, ainda, a redução da força periférica, facilitando assim a queda ${ }^{1}$.

A queda é definida como um evento não intencional, no qual o sujeito sofre uma mudança abrupta de uma posição para um nível abaixo em relação a sua posição inicial. A ocorrência de quedas aumenta com o avançar da idade, pressupondo que cerca de $34 \%$ dos idosos entre 65 e 80 anos, $45 \%$ entre 80 a 89 anos e $50 \%$ acima dos 90 anos sofrerão com algum episódio durante a sua vida ${ }^{3}$.

Alguns fatores colaboram com a maior vulnerabilidade observada em idosos, como, idade avançada ( $\geq 65$ anos), gênero feminino, pós-operatório, doenças crônicas, hipotensão ortostática, diminuição da força muscular, dificuldade de deambular, déficit cognitivo, histórico de quedas, polifarmácia contínua e uso de benzodiazepínicos e anti-hipertensivos ${ }^{4,5}$. $\mathrm{O}$ uso desses medicamentos está entre um dos diagnósticos de admissão hospitalar, sendo um dos motivos que mantêm por mais tempo o sujeito internado.

Mesmo em idosos sem alterações para realização das atividades de vida diária (AVD), o avanço da idade está relacionado à diminuição da mobilidade e da força muscular ${ }^{6}$. Nesse contexto, a mensuração da força de preensão palmar é sugerida pela literatura como uma medida clínica simples no rastreamento e na identificação de modificações funcionais pequenas, devido à sua associação com a função muscular de membros inferiores? ${ }^{7}$.

A internação hospitalar ou em instituições de longa permanência colabora com a piora da funcionalidade e, consequentemente, propicia o episódio da queda, o que se evidencia em uma comparação com idosos que vivem na comunida$\mathrm{de}^{8}$. A hospitalização poderá promover o declínio funcional do indivíduo, o qual é justificado por: idade avançada, diagnóstico de entrada, repouso excessivo, privação do sono, perda da autonomia, déficit cognitivo e polifarmácia, além de estar, nessa situação, favorecida uma maior probabilidade de desencadear outras comorbidades ${ }^{1}$.

Um dos principais objetivos da gerontologia é o cuidado com idosos e a manutenção da sua independência e de sua autonomia, promovendo a qualidade de vida para esses indivíduos. Estudar a relação entre as quedas e a capacidade funcional pode ser fator preditivo para a qualidade de vida dessa população9. Além disso, relacionar tais fatores à força muscular pode ser um diferencial no momento de avaliar o idoso hospitalizado para compreender com maior clareza seu estado clínico-funcional e delinear o melhor tratamento e o manejo para a alta hospitalar. Nesse sentido, o presente estudo objetivou avaliar o risco de quedas, a força muscular periférica e a capacidade funcional de idosos hospitalizados.

\section{Métodos}

A pesquisa é de caráter transversal. O estudo ocorreu no Hospital da Cidade de Passo Fundo/ RS - Atualmente, Hospital de Clínicas de Passo Fundo -, durante o período de junho a julho de 2017, participaram da pesquisa 100 idosos hospitalizados com idade entre 65 e 80 anos. Para todos os sujeitos, foi dada uma explicação detalhada da 
pesquisa, para aqueles que aceitaram participar foi fornecida uma cópia do Termo de Consentimento Livre e Esclarecido (TCLE). Foram excluídos os indivíduos que apresentassem impossibilidade de flexão dos dedos, incapacidade de sair do leito e/ ou de deambulação, ainda aqueles que manifestassem sintomas de intolerância durante os testes ou indivíduos que não conseguissem responder as escalas. O projeto foi aprovado pelo Comitê de Ética de Pesquisa da Universidade de Passo Fundo sob o número 2.114.541.

A avaliação inicial era composta pela coleta de dados que envolveu as seguintes variáveis: idade, gênero, dados antropométricos, dados específicos sobre a doença, patologias associadas, medicamentos em uso, mensuração da força muscular periférica, equilíbrio e mobilidade, risco de quedas e funcionalidade. Foram realizados os testes de Kendall, "Timed Up And Go" (TUG), Dinamometria manual, Escala de Morse e Escala PS-ECOG.

Teste de Kendall: avalia o grau de força muscular exercido durante o movimento em determinada área, sendo medido em $\mathrm{kg} / \mathrm{f}$. Foram avaliados os seguintes grupos musculares: flexores, extensores, adutores e abdutores de ombro; flexores, extensores, pronadores e supinadores de cotovelo; flexores e extensores de punho; flexores, extensores, adutores e abdutores de quadril; flexores e extensores de joelho, e flexores e extensores de tornozelo. O grau de força muscular é definido com uma escala numérica de zero a dez, sendo zero para quando não há contração muscular visível e dez para quando a articulação move-se por meio de um arco de movimento completo, contra pressão máxima. Por meio da classificação numérica, são descritos os graus de força muscular, sendo classificados na seguinte escala: normal, bom, regular, fraco e zero, para forte e moderada força, resistência contra a gravidade, movimento a favor da gravidade, e sem visualização de contração, respectivamente ${ }^{10}$.

Dinamometria manual: os idosos realizaram o teste de força de preensão palmar por meio do dinamômetro "Kratos", um instrumento que mede a intensidade de força de preen- são produzida por uma contração isométrica, o resultado foi estimado em $\mathrm{kg} / \mathrm{f}$. Os indivíduos foram posicionados sentados em cadeira padronizada, ombros abduzidos, cotovelos fletidos a $90^{\circ}$, antebraço e punho em posição neutra. Os participantes deveriam exercer força máxima, aplicando pressão na alavanca contra a base do dinamômetro, até que o ponteiro atingisse um pico. A avaliação foi iniciada pela mão dominante, sendo realizadas três medidas com duração de cinco segundos de contração cada, o intervalo de tempo para cada membro foi de um minuto, a fim de não provocar fadiga muscular durante o teste, foi considerado o maior valor obtido entre as tentativas. O examinador sustentava o dinamômetro, enquanto realizava um estímulo auditivo para que o sujeito conseguisse contração voluntária máxima ${ }^{7}$.

TUG: o teste quantifica em segundos a mobilidade funcional, tendo por objetivo avaliar o risco de queda do sujeito. Para a realização desse, solicitou-se ao indivíduo que ele levantasse de uma cadeira sem se apoiar nas laterais, caminhasse três metros e retornasse à cadeira. $\mathrm{O}$ tempo era medido por um cronômetro a partir da ordem de "vá". Para valores de tempo menores de dez segundos, estipulou-se que esses indivíduos apresentavam baixo risco de quedas, sendo considerados com mobilidade normal; os que necessitassem de 11 a 20 segundos foram classificados como indivíduos frágeis, porém independentes e com boa mobilidade, manifestando baixo risco de queda; aqueles participantes que precisaram de 21 a 30 segundos para percorrer o trajeto foram classificados como indivíduos com moderado risco de queda, sendo considerados semidependentes com mobilidade regular; aqueles sujeitos que necessitaram acima de 30 segundos para a realização do teste, foi dada a classificação de elevado risco de quedas, manifestando mobilidade prejudicada, sendo apontados como dependentes ${ }^{7,11}$.

Escala de Morse: a escala avalia em porcentagem o risco de queda por intermédio de seis itens, a pontuação é dada dependendo da resposta do indivíduo. Apresentamos a seguir 
os itens: história anterior de queda: não caiu nos últimos três meses: zero ponto. Sim, caiu nos últimos três meses: 25 pontos. Existência de um diagnóstico secundário: não possui mais de um diagnóstico secundário: zero ponto. Sim, possui mais de um diagnóstico secundário: 15 pontos. Auxílio na deambulação: não utiliza/ totalmente acamado/ deambula auxiliado com profissional de saúde: zero ponto. Usa muleta/ bengala/andador: 15 pontos. Segura no mobiliário/parede: 30 pontos. Terapia intravenosa/ dispositivo endovenoso salinizado ou heparinizado: Não faz uso: zero ponto. Sim, faz uso: 20 pontos. Marcha: normal/não deambula/totalmente acamado/uso de cadeiras de rodas: zero ponto. Marcha fraca: dez pontos. Marcha comprometida/cambaleante: 20 pontos. Estado mental: Orientado/capaz quanto à sua capacidade/ limitação: zero ponto. Superestima capacidade/ esquece limitações: 15 pontos. A pontuação total da escala varia entre zero e 125 , os indivíduos são distinguidos pela somatória dos valores apresentando baixo risco de queda para pontuação entre zero-24 pontos, risco moderado entre 25-44 pontos e risco elevado de queda $\geq 45^{12}$.

Escala PS-ECOG: esta escala foi utilizada para avaliar a capacidade funcional dos idosos. Esse instrumento avalia como a patologia afeta as habilidades de vida diária do paciente, sendo o resultado estimado em porcentagem, a pontuação varia de zero a cinco, classificando o sujeito em: índice zero - assintomático: totalmente ativo, sem restrição; um - sintomático: restritos para atividade física extenuante, porém capazes de realizar um trabalho de natureza leve ou sedentária; dois - sintomático: completamente capaz para o autocuidado, mas incapaz de realizar quaisquer atividades de trabalho, estando fora do leito por mais de $50 \%$ do tempo; três - sintomático: capacidade de autocuidado limitada, restrito ao leito ou à cadeira por mais da metade do tempo; quatro - acamado: completamente limitado, restrito ao leito ou à cadeira, sem poder exercer qualquer atividade de autocuidado; cinco morto ${ }^{13}$.

Por meio de um software estatístico, as variáveis foram expressas por meio de estatística descritivas em que apresentaram-se os valores das variáveis numéricas em média e desvio padrão e das variáveis nominais em frequência absoluta e relativa. Ainda, utilizou-se o teste $\mathrm{t}$ de amostras independentes para calcular o cruzamento entre as variáveis numéricas e as variáveis dependentes "risco de quedas" e "capacidade funcional" e o teste Qui-Quadrado para calcular o cruzamento entre as variáveis nominais e as variáveis dependentes "risco de quedas" e "capacidade funcional".

\section{Resultados}

Para a realização do estudo foram avaliados 100 idosos hospitalizados, sendo a amostra composta por 43 (43\%) indivíduos do gênero feminino e 57 (57\%) pertencentes ao gênero mas-

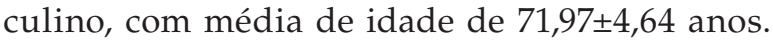
Percebe-se que os indivíduos possuíam IMC e PAS e PAD de repouso dentro da normalidade. Nota-se ainda, a baixa adesão física entre os participantes, sendo praticada apenas por $20 \%$ dos investigados, destaca-se que todos os idosos faziam uso de múltiplos medicamentos, entre eles, os anti-hipertensivos e os benzodiazepínicos, que estão relacionados à pré-disposição à queda. Em relação ao Teste TUG, a maioria da amostra foi classificada como frágil, porém independente e com boa mobilidade, manifestando baixo risco de queda (Tabela 1).

A Tabela 2 representa a média de força muscular periférica durante os movimentos articulares das articulações dos ombros, cotovelos, punhos, quadris, joelhos e tornozelos, verificada por meio da escala Kendall, e das mãos, verificada por meio da dinamometria manual. Em todas as articulações periféricas avaliadas por meio da escala Kendall, os indivíduos apresentaram força muscular entre 4,08 kg/f e 4,62 kg/f (média geral de $4,47 \mathrm{~kg} / \mathrm{f}$ ), classificando-os com uma intensidade regular. Em relação à força muscular da preensão das mãos, os indivíduos apresentaram no membro direito a média de $17,72 \mathrm{~kg} / \mathrm{f}$ e no membro esquerdo média de 17,33 kg/f. 
Tabela l: Caracterização da amostra. Passo Fundo/RS, 2017

\begin{tabular}{c|c}
\hline Variável & Frequência (\%) \\
\hline Gênero masculino & $57(57 \%)$ \\
\hline Idade (anos) & $71,97 \pm 4,64$ \\
\hline Índice de massa corporal $\left(\mathrm{kg} / \mathrm{m}^{2}\right)$ & $24,78 \pm 4,20$ \\
\hline Pressão arterial sistólica $(\mathrm{mmHg})$ & $128,3 \pm 14,97$ \\
\hline Pressão arterial diastólica $(\mathrm{mmHg})$ & $80,6 \pm 9,40$ \\
\hline Não pratica de atividade física & $80(80 \%)$ \\
\hline Uso de polifarmácia & $100(100 \%)$ \\
\hline Uso de anti-hipertensivo & $97(97 \%)$ \\
\hline Uso de benzodiazepínicos & $70(70 \%)$ \\
\hline Timed Up And Go Test (segundos) & $12,25 \pm 9,13$ \\
\hline
\end{tabular}

Fonte: os próprios autores

Tabela 2: Força muscular periférica. Passo Fundo/RS, 2017

\begin{tabular}{c|c|c|c|c}
\hline \multicolumn{2}{c}{ Variável Direito } & \multicolumn{2}{c}{ Variável Esquerdo } \\
\hline & Movimento & $\begin{array}{c}\text { Resultados } \\
\text { FM (Kg/f) }\end{array}$ & Movimento & $\begin{array}{c}\text { Resultados } \\
\text { FM (Kg/f) }\end{array}$ \\
\hline \multirow{4}{*}{ Ombro } & Flexão & $4,58 \pm 0,62$ & Flexão & $4,58 \pm 0,62$ \\
& Extensão & $4,59 \pm 0,62$ & Extensão & $4,59 \pm 0,62$ \\
& Abdução & $4,59 \pm 0,62$ & Abdução & $4,59 \pm 0,62$ \\
& Adução & $4,59 \pm 0,62$ & Adução & $4,59 \pm 0,62$ \\
& Abdução horizontal & $4,59 \pm 0,62$ & Abdução horizontal & $4,59 \pm 0,62$ \\
& Adução horizontal & $4,59 \pm 0,62$ & Adução horizontal & $4,59 \pm 0,62$ \\
\hline \multirow{4}{*}{ Cotovelo } & Flexão & $4,61 \pm 0,58$ & Flexão & $4,61 \pm 0,58$ \\
& Extensão & $4,62 \pm 0,58$ & Extensão & $4,62 \pm 0,58$ \\
& Pronação & $4,61 \pm 0,58$ & Pronação & $4,61 \pm 0,58$ \\
& Supinação & $4,61 \pm 0,58$ & Supinação & $4,61 \pm 0,58$ \\
\hline \multirow{2}{*}{ Punho } & Flexão & $4,62 \pm 0,54$ & Flexão & $4,62 \pm 0,54$ \\
& Extensão & $4,62 \pm 0,54$ & Extensão & $4,62 \pm 0,54$ \\
\hline \multirow{3}{*}{ Quadril } & Flexão & $4,08 \pm 0,84$ & Flexão & $4,08 \pm 0,84$ \\
& Extensão & $4,08 \pm 0,84$ & Extensão & $4,08 \pm 0,84$ \\
& Abdução & $4,09 \pm 0,84$ & Abdução & $4,09 \pm 0,84$ \\
& Adução & $4,09 \pm 0,84$ & Adução & $4,09 \pm 0,84$ \\
\hline \multirow{2}{*}{ Joelho } & Flexão & $4,42 \pm 0,63$ & Flexão & $4,43 \pm 0,63$ \\
& Extensão & $4,43 \pm 0,63$ & Extensão & $4,43 \pm 0,63$ \\
\hline \multirow{2}{*}{ Mornozelo } & Plantiflexão & $4,48 \pm 0,61$ & Plantiflexão & $4,48 \pm 0,61$ \\
& Dorsiflexão & $4,48 \pm 0,61$ & Dorsiflexão & $4,48 \pm 0,61$ \\
\hline & Força de preensão & $17,72 \pm 8,69$ & Força de preensão & $17,33 \pm 8,34$ \\
\hline
\end{tabular}

Legenda: Kg/F: quilogramas-força.

Fonte: os próprios autores.

Em relação aos resultados encontrados na escala de Morse Fall Scale (MFS), verificou-se que $77 \%$ indivíduos negaram histórico de queda, 92\% possuíam algum diagnóstico secundário, $44 \%$ faziam uso de terapia intravenosa, $89 \%$ sujeitos confirmaram necessitar de auxílio durante a deambulação, sendo que para $90 \%$ dos participantes a marcha foi considerada normal, e $96 \%$ dos idosos estavam orientados sobre sua atual situação, capacidades e limitações. Identificouse que a maioria dos indivíduos apresentou risco moderado de quedas. A em relação à capacidade funcional, de acordo com a pontuação obtida pela escala PS-ECOG, a maioria dos indivíduos obteve pontuação um, a qual refere-se à restrição dos sujeitos para atividades físicas extenuantes, porém com capacidade de realizar atividades leves ou de natureza sedentária. (Tabela 3)

Observou-se que os fatores relacionados ao moderado ou ao elevado risco de quedas nos idosos foram o aumento do tempo para realizar o Teste TUG e o maior impacto sobre a capacidade funcional (pontuação no escores dois, três, quatro ou cinco da escala PSECOG). (Tabela 4)

Observou-se que os fatores relacionados ao maior impacto da capacidade funcional nos idosos foram o aumento da idade, a menor força muscular periférica em todas as articulações avaliadas pela escala Kendall e o moderado ou o elevado risco de quedas. (Tabela 5)

\section{Discussão}

Com o aumento da população idosa, paralelamente cresce o número de admissões hospitalares. É sabido que a hospitalização acarreta redução da capacidade funcional, interferindo a qualidade de vida dos indivíduos, além de abrir portas para outras comorbidades ${ }^{8}$. Durante esse período, o idoso apresenta um aumento de fragilidade devido à patologia de origem e à perda da autonomia, esse declínio da funcionalidade poderá dificultar ou impedir a execução de tarefas, provocando repercussões durante a internação e após a alta hospitalar ${ }^{14}$. 
Tabela 3: Escala de quedas Morse e escala PS-ECOG. Passo Fundo/RS, 2017

\begin{tabular}{|c|c|}
\hline Variáveis & Frequência \% \\
\hline \multicolumn{2}{|c|}{ Escala de Risco de Quedas de Morse } \\
\hline Baixo risco de quedas & $42 \%$ \\
\hline Moderado risco de quedas & $44 \%$ \\
\hline Elevado risco de quedas & $14 \%$ \\
\hline \multicolumn{2}{|l|}{ Escala PS-ECOG } \\
\hline 0 & $22 \%$ \\
\hline 1 & $36 \%$ \\
\hline 2 & $7 \%$ \\
\hline 3 & $15 \%$ \\
\hline 4 & $15 \%$ \\
\hline 5 & $5 \%$ \\
\hline
\end{tabular}

Fonte: próprios autores
Um estudo que analisou o perfil de idosos internados em ambiente hospitalar demonstrou que a maioria da amostra se concentrava na faixa etária dos 60-69 anos e era do gênero feminino $^{15}$. Tais dados vão de encontro aos nossos resultados onde observamos que a maioria da amostra era do gênero masculino com media de idade de $71,97 \pm 4,64$.

Visando à manutenção da funcionalidade no idoso, sabe-se que o exercício físico promove bem-estar físico, social e mental, ou seja, a prática regular de atividade física prepara o longevo para uma vida autônoma e independente. Contudo, em indivíduos sedentários ocorre uma maior acentuação no declínio da capacidade

Tabela 4: Relação entre o risco de quedas da escala Morse e as varióveis sociodemográficas, a força muscular periférica e a capacidade funcional. Passo Fundo/RS, 2017

\begin{tabular}{lccc}
\hline \multicolumn{1}{c}{ Variáveis } & $\begin{array}{c}\text { Baixo risco de quedas } \\
(\mathrm{n}=42)\end{array}$ & $\begin{array}{c}\text { Moderado ou elevado } \\
\text { risco de quedas } \\
(\mathrm{n}=58)\end{array}$ & Valor $\mathbf{p}$ \\
\hline Sociodemográficas & & & \\
\hline Sexo & $18(41,9 \%)$ & $25(58,1 \%)$ & 1,000 \\
Feminino & $24(42,1 \%)$ & $33(57,9 \%)$ & \\
Masculino & $71,57 \pm 4,32$ & $72,26 \pm 4,88$ & 0,468 \\
\hline Idade & $24,17 \pm 4,70$ & $25,22 \pm 3,79$ & 0,224 \\
\hline IMC & $09(45,0 \%)$ & $11(55,0 \%)$ & 0,804 \\
\hline Atividade física & $33(41,2 \%)$ & $47(58,8 \%)$ & \\
Sim & $9,85 \pm 6,08$ & $13,99 \pm 10,53$ & $0,015 *$ \\
\hline Não & Força muscular periférica & & \\
\hline Tempo do TUG Test & $4,69 \pm 0,51$ & $4,50 \pm 0,68$ & 0,116 \\
\hline & $4,69 \pm 0,51$ & $4,50 \pm 0,68$ & 0,116 \\
\hline Flexão ombro direito & $4,69 \pm 0,51$ & $4,52 \pm 0,68$ & 0,152 \\
\hline Flexão ombro esquerdo & $4,69 \pm 0,51$ & $4,52 \pm 0,68$ & 0,152 \\
\hline Extensão ombro direito & $4,69 \pm 0,51$ & $4,52 \pm 0,68$ & 0,152 \\
\hline Extensão ombro esquerdo & $4,69 \pm 0,51$ & $4,52 \pm 0,68$ & 0,152 \\
\hline Adução ombro direito & $4,69 \pm 0,51$ & $4,52 \pm 0,68$ & 0,152 \\
\hline Adução ombro esquerdo & $4,69 \pm 0,51$ & $4,52 \pm 0,68$ & 0,152 \\
\hline Abdução ombro direito & $4,69 \pm 0,51$ & $4,52 \pm 0,68$ & 0,152 \\
\hline Abdução ombro esquerdo & $4,69 \pm 0,51$ & $4,52 \pm 0,68$ & 0,152 \\
\hline Adução horizontal ombro direito & $4,69 \pm 0,51$ & $4,52 \pm 0,68$ & 0,152 \\
\hline Adução horizontal ombro esquerdo & & & \\
\hline Abdução horizontal ombro direito & & \\
\hline
\end{tabular}

Legenda: TUG: Timed Up And Go; ${ }^{*}(p<0,05)$.

Fonte: os próprios autores. 
Continuação - Tabela 4: Relação entre o risco de quedas da escala Morse e as varióveis sociodemográficas, a força muscular periférica e a capacidade funcional. Passo Fundo/RS, 2017

\begin{tabular}{|c|c|c|c|}
\hline Variáveis & $\begin{array}{l}\text { Baixo risco de quedas } \\
\qquad(\mathrm{n}=42)\end{array}$ & $\begin{array}{l}\text { Moderado ou elevado } \\
\text { risco de quedas } \\
(n=58)\end{array}$ & Valor $\mathrm{p}$ \\
\hline Abdução horizontal ombro esquerdo & $4,69 \pm 0,51$ & $4,52 \pm 0,68$ & 0,152 \\
\hline Flexão cotovelo direito & $4,71 \pm 0,45$ & $4,53 \pm 0,65$ & 0,109 \\
\hline Flexão cotovelo esquerdo & $4,71 \pm 0,45$ & $4,53 \pm 0,65$ & 0,109 \\
\hline Extensão cotovelo direito & $4,71 \pm 0,45$ & $4,55 \pm 0,65$ & 0,147 \\
\hline Extensão cotovelo esquerdo & $4,71 \pm 0,45$ & $4,55 \pm 0,65$ & 0,147 \\
\hline Pronação cotovelo direito & $4,71 \pm 0,45$ & $4,53 \pm 0,65$ & 0,109 \\
\hline Pronação cotovelo esquerdo & $4,71 \pm 0,45$ & $4,53 \pm 0,65$ & 0,109 \\
\hline Supinação cotovelo direito & $4,71 \pm 0,45$ & $4,53 \pm 0,65$ & 0,109 \\
\hline Supinação cotovelo esquerdo & $4,71 \pm 0,45$ & $4,53 \pm 0,65$ & 0,109 \\
\hline Flexão punho direito & $4,69 \pm 0,46$ & $4,57 \pm 0,59$ & 0,256 \\
\hline Flexão punho esquerdo & $4,69 \pm 0,46$ & $4,57 \pm 0,59$ & 0,256 \\
\hline Extensão punho direito & $4,69 \pm 0,46$ & $4,57 \pm 0,59$ & 0,256 \\
\hline Extensão punho esquerdo & $4,69 \pm 0,46$ & $4,57 \pm 0,59$ & 0,256 \\
\hline Flexão joelho direito & $4,48 \pm 0,63$ & $4,38 \pm 0,64$ & 0,456 \\
\hline Flexão joelho esquerdo & $4,50 \pm 0,63$ & $4,38 \pm 0,64$ & 0,353 \\
\hline Extensão joelho direito & $4,50 \pm 0,63$ & $4,38 \pm 0,64$ & 0,353 \\
\hline Extensão joelho esquerdo & $4,50 \pm 0,63$ & $4,38 \pm 0,64$ & 0,353 \\
\hline Plantiflexão tornozelo direito & $4,55 \pm 0,55$ & $4,43 \pm 0,65$ & 0,336 \\
\hline Plantiflexão tornozelo esquerdo & $4,55 \pm 0,55$ & $4,43 \pm 0,65$ & 0,336 \\
\hline Dorsiflexão tornozelo direito & $4,55 \pm 0,55$ & $4,43 \pm 0,65$ & 0,336 \\
\hline Dorsiflexão tornozelo esquerdo & $4,55 \pm 0,55$ & $4,43 \pm 0,65$ & 0,336 \\
\hline Flexão quadril direito & $4,21 \pm 0,89$ & $3,98 \pm 0,80$ & 0,188 \\
\hline Flexão quadril esquerdo & $4,21 \pm 0,89$ & $3,98 \pm 0,80$ & 0,188 \\
\hline Extensão quadril direito & $4,21 \pm 0,89$ & $3,98 \pm 0,80$ & 0,188 \\
\hline Extensão quadril esquerdo & $4,21 \pm 0,89$ & $3,98 \pm 0,80$ & 0,188 \\
\hline Adução quadril direito & $4,21 \pm 0,89$ & $4,00 \pm 0,79$ & 0,220 \\
\hline Adução quadril esquerdo & $4,21 \pm 0,89$ & $4,00 \pm 0,79$ & 0,220 \\
\hline Abdução quadril direito & $4,21 \pm 0,89$ & $4,00 \pm 0,79$ & 0,220 \\
\hline Abdução quadril esquerdo & $4,21 \pm 0,89$ & $4,00 \pm 0,79$ & 0,220 \\
\hline Força de preensão da mão direita & $18,54 \pm 7,72$ & $17,13 \pm 9,35$ & 0,413 \\
\hline Força de preensão da mão esquerda & $18,19 \pm 8,78$ & $16,71 \pm 8,03$ & 0,393 \\
\hline Capacidade funcional & & & $0,025^{*}$ \\
\hline Menor impacto na capacidade funcional & $30(51,7 \%)$ & $28(48,3 \%)$ & \\
\hline Maior impacto na capacidade funcional & $12(28,6 \%)$ & $30(71,4 \%)$ & \\
\hline
\end{tabular}

Legenda: TUG: Timed Up And Go; ${ }^{*}(\mathrm{p}<0,05)$.

Fonte: os próprios autores.

funcional e, consequentemente, há um declive na qualidade de vida ${ }^{16,17}$. Outro fator que corrobora com consecutivas internações hospitalares é o alto índice de fármacos administrados entre os participantes ${ }^{1}$. A polifarmácia pode estar relacionada a diversos desfechos indesejáveis à saúde como a diminuição da capacidade funcional, o declínio cognitivo e o risco de eventos adversos devido a interações medicamentosas, como, também, ao aumento do risco de queda 
Tabela 5: Relação entre a capacidade funcional e as variớveis sociodemográficas, a força muscular periférica e o risco de quedas. Passo Fundo/RS, 2017

\begin{tabular}{|c|c|c|c|}
\hline Variáveis & $\begin{array}{l}\text { Menor impacto na } \\
\text { capacidade funcional } \\
(n=58)\end{array}$ & $\begin{array}{l}\text { Maior impacto na } \\
\text { capacidade funcional } \\
(\mathrm{n}=42)\end{array}$ & Valor $\mathrm{p}$ \\
\hline \multicolumn{4}{|c|}{ Sociodemográficas } \\
\hline Sexo & & & 0,540 \\
\hline Feminino & $23(53,5 \%)$ & $20(46,5 \%)$ & \\
\hline Masculino & $35(61,4 \%)$ & $22(38,6 \%)$ & \\
\hline Idade & $71,00 \pm 4,23$ & $73,31 \pm 4,90$ & $0,013^{*}$ \\
\hline $\mathrm{IMC}$ & $24,86 \pm 4,05$ & $24,66 \pm 4,46$ & 0,815 \\
\hline Atividade física & & & 0,455 \\
\hline Sim & $10(50,0 \%)$ & $10(50,0 \%)$ & \\
\hline Não & $48(60,0 \%)$ & $32(40,0 \%)$ & \\
\hline Tempo do TUG Test & $12,17 \pm 7,09$ & $12,35 \pm 11,46$ & 0,929 \\
\hline \multicolumn{4}{|c|}{ Força muscular periférica } \\
\hline Flexão ombro direito & $4,72 \pm 0,52$ & $4,38 \pm 0,69$ & $0,009^{*}$ \\
\hline Flexão ombro esquerdo & $4,72 \pm 0,52$ & $4,38 \pm 0,69$ & $0,009^{*}$ \\
\hline Extensão ombro direito & $4,74 \pm 0,51$ & $4,38 \pm 0,69$ & $0,006^{*}$ \\
\hline Extensão ombro esquerdo & $4,74 \pm 0,51$ & $4,38 \pm 0,69$ & $0,006^{*}$ \\
\hline Adução ombro direito & $4,74 \pm 0,51$ & $4,38 \pm 0,69$ & $0,006^{*}$ \\
\hline Adução ombro esquerdo & $4,74 \pm 0,51$ & $4,38 \pm 0,69$ & $0,006^{*}$ \\
\hline Abdução ombro direito & $4,74 \pm 0,51$ & $4,38 \pm 0,69$ & $0,006^{*}$ \\
\hline Abdução ombro esquerdo & $4,74 \pm 0,51$ & $4,38 \pm 0,69$ & $0,006^{*}$ \\
\hline Adução horizontal ombro direito & $4,74 \pm 0,51$ & $4,38 \pm 0,69$ & $0,006^{*}$ \\
\hline Adução horizontal ombro esquerdo & $4,74 \pm 0,51$ & $4,38 \pm 0,69$ & $0,006^{*}$ \\
\hline Abdução horizontal ombro direito & $4,74 \pm 0,51$ & $4,38 \pm 0,69$ & $0,006^{*}$ \\
\hline Abdução horizontal ombro esquerdo & $4,74 \pm 0,51$ & $4,38 \pm 0,69$ & $0,006^{*}$ \\
\hline Flexão cotovelo direito & $4,76 \pm 0,47$ & $4,40 \pm 0,66$ & $0,004^{*}$ \\
\hline Flexão cotovelo esquerdo & $4,76 \pm 0,47$ & $4,40 \pm 0,66$ & $0,004^{*}$ \\
\hline Extensão cotovelo direito & $4,78 \pm 0,46$ & $4,40 \pm 0,66$ & $0,003^{*}$ \\
\hline Extensão cotovelo esquerdo & $4,78 \pm 0,46$ & $4,40 \pm 0,66$ & $0,003^{*}$ \\
\hline Pronação cotovelo direito & $4,76 \pm 0,47$ & $4,40 \pm 0,66$ & $0,004^{*}$ \\
\hline Pronação cotovelo esquerdo & $4,76 \pm 0,47$ & $4,40 \pm 0,66$ & $0,004^{*}$ \\
\hline Supinação cotovelo direito & $4,76 \pm 0,47$ & $4,40 \pm 0,66$ & $0,004^{*}$ \\
\hline Supinação cotovelo esquerdo & $4,76 \pm 0,47$ & $4,40 \pm 0,66$ & $0,004^{*}$ \\
\hline Flexão punho direito & $4,74 \pm 0,48$ & $4,45 \pm 0,59$ & $0,011^{*}$ \\
\hline Flexão punho esquerdo & $4,74 \pm 0,48$ & $4,45 \pm 0,59$ & $0,011^{*}$ \\
\hline Extensão punho direito & $4,74 \pm 0,48$ & $4,45 \pm 0,59$ & $0,011^{*}$ \\
\hline Extensão punho esquerdo & $4,74 \pm 0,48$ & $4,45 \pm 0,59$ & $0,011^{*}$ \\
\hline Flexão joelho direito & $4,59 \pm 0,59$ & $4,19 \pm 0,63$ & $0,002^{*}$ \\
\hline Flexão joelho esquerdo & $4,60 \pm 0,59$ & $4,19 \pm 0,63$ & $0,001^{*}$ \\
\hline Extensão joelho direito & $4,60 \pm 0,59$ & $4,19 \pm 0,63$ & $0,001^{*}$ \\
\hline Extensão joelho esquerdo & $4,60 \pm 0,59$ & $4,19 \pm 0,63$ & $0,001^{*}$ \\
\hline Plantiflexão tornozelo direito & $4,64 \pm 0,55$ & $4,26 \pm 0,62$ & $0,002^{*}$ \\
\hline Plantiflexão tornozelo esquerdo & $4,64 \pm 0,55$ & $4,26 \pm 0,62$ & $0,002^{*}$ \\
\hline
\end{tabular}

Legenda: TUG: Timed Up And Go; ${ }^{*}(p<0,05)$.

Fonte: os próprios autores. 
Continuação - Tabela 5: Relação entre a capacidade funcional e as varióveis sociodemográficas, a força muscular periférica e o risco de quedas. Passo Fundo/RS, 2017

\begin{tabular}{|c|c|c|c|}
\hline Variáveis & $\begin{array}{l}\text { Menor impacto na } \\
\text { capacidade funcional } \\
\qquad(\mathrm{n}=58)\end{array}$ & $\begin{array}{l}\text { Maior impacto na } \\
\text { capacidade funcional } \\
(\mathrm{n}=42)\end{array}$ & Valor $\mathrm{p}$ \\
\hline Dorsiflexão tornozelo direito & $4,64 \pm 0,55$ & $4,26 \pm 0,62$ & $0,002^{*}$ \\
\hline Dorsiflexão tornozelo esquerdo & $4,64 \pm 0,55$ & $4,26 \pm 0,62$ & $0,002^{*}$ \\
\hline Flexão quadril direito & $4,31 \pm 0,82$ & $3,76 \pm 0,79$ & $0,001^{*}$ \\
\hline Flexão quadril esquerdo & $4,31 \pm 0,82$ & $3,76 \pm 0,79$ & $0,001^{*}$ \\
\hline Extensão quadril direito & $4,31 \pm 0,82$ & $3,76 \pm 0,79$ & $0,001^{*}$ \\
\hline Extensão quadril esquerdo & $4,31 \pm 0,82$ & $3,76 \pm 0,79$ & $0,001^{*}$ \\
\hline Adução quadril direito & $4,31 \pm 0,82$ & $3,79 \pm 0,78$ & $0,002^{*}$ \\
\hline Adução quadril esquerdo & $4,31 \pm 0,82$ & $3,79 \pm 0,78$ & $0,002^{*}$ \\
\hline Abdução quadril direito & $4,31 \pm 0,82$ & $3,79 \pm 0,78$ & $0,002^{*}$ \\
\hline Abdução quadril esquerdo & $4,31 \pm 0,82$ & $3,79 \pm 0,78$ & $0,002^{*}$ \\
\hline Força de preensão da mão direita & $19,03 \pm 8,41$ & $15,91 \pm 8,84$ & 0,077 \\
\hline Força de preensão da mão esquerda & $18,59 \pm 8,81$ & $15,59 \pm 7,40$ & 0,076 \\
\hline Risco de Quedas & & & $0,025^{*}$ \\
\hline Baixo risco & $30(71,4 \%)$ & $12(28,6 \%)$ & \\
\hline Moderado ou elevado risco & $28(48,3 \%)$ & $30(51,7 \%)$ & \\
\hline
\end{tabular}

Legenda: TUG: Timed Up And Go; * $(p<0,05)$.

Fonte: os próprios autores.

afetando a autonomia e principalmente na qualidade de vida ${ }^{18}$. Esse fato pode ter contribuído para a maioria da amostra ter apresentado moderado ou elevado risco de quedas e algum grau de impacto em sua capacidade funcional (que pontuaram nos escores um, dois, três, quatro ou cinco da escala PS-ECOG). Embora não tenha sido possível analisar estatisticamente a relação entre o uso de polifarmácia e as variáveis independentes, em virtude de $100 \%$ da amostra fazer uso deste componente, sugere-se que em futuros estudos estude-se tais relações.

Dentre os principais medicamentos que têm o potencial de alterar a estabilidade corporal estão o uso de benzodiazepínicos e anti-hipertensivos. Os fármacos administrados de modo inadequado pelos idosos são capazes comprometer sua funcionalidade, pré-dispondo-o ao risco de queda, podendo causar sedação, comprometimento do equilíbrio e da coordenação, além de poder agravar a hipotensão ortostática, diminuir as funções motoras incluindo o tempo de reação, gerando fadiga e vertigem. Entende-se que o idoso é mais frágil que o adulto jovem, portanto, mais suscetível aos efeitos adversos das drogas administradas $^{19,20}$. Nesse sentido, atentamos para o fato de que nossa amostra tenha apresentado altos índices de uso dos fármacos supracitados ( $97 \%$ antihipertensivos e 70\% benzodiazepínicos).

Em relação ao teste de TUG, foi verificado que os indivíduos participantes do estudo apresentam independência funcional. Ao encontro, uma pesquisa composta por 99 idosos, com idade média de 68,3 anos, que teve por objetivo avaliar a dor lombar e a capacidade funcional por meio do teste Timed Up And Go Test, classificou 66\% desses idosos como independentes e sem risco de queda ${ }^{21}$. Ao contrário, no estudo de Pereira et al. ${ }^{13}$ que avaliou 94 idosos, entre os domínios de avaliação da capacidade funcional global, a avaliação cognitiva, a avaliação do humor, a avaliação da funcionalidade da comunicação e a avaliação da mobilidade, por meio do teste de TUG, os autores encontraram um desempenho médio entre os participantes, classificando-os com uma mobilidade regular ou prejudicada. 
Conforme o indivíduo envelhece, ocorre a perda da massa muscular esquelética, especialmente após os 50 anos, chegando por vezes, a ser percebido um decréscimo de $8 \%$ a $15 \%$ por década após 22. Em relação à força muscular periférica, através da escala Kendall, obtivemos nesta pesquisa força muscular graduada de intensidade regular em todas as articulações avaliadas, com valores que variaram entre $4,08 \mathrm{~kg} / \mathrm{f}$ e 4,62 $\mathrm{kg} / \mathrm{f}$ (média geral de 4,47 kg/f). Esse resultado é apoiado pela revisão bibliográfica de Vieira et al. ${ }^{22}$, na qual os autores apontam que a redução da massa e da força muscular é observada facilmente em idosos. Essa fraqueza acomete com maior intensidade os membros inferiores, levando à perda do equilíbrio estático e dinâmico, favorecendo o risco de quedas, fraturas, internações e até mesmo de morte. Ainda que essa perda da força muscular seja multifatorial, sabe-se que existem alguns preditores que contribuem para que ocorra com maior acentuação, tais como, diminuição dos níveis de atividade física, alimentação deficitária, alteração hormonal e do sistema nervoso central.

O declínio da força muscular também pode ser notado em relação à força de preensão palmar, como verificado neste estudo, pois, de acordo com a literatura, os pontos de corte são de $20 \mathrm{~kg}$ para mulheres e $30 \mathrm{~kg}$ para homens ${ }^{23}$. Uma pesquisa realizada com 404 indivíduos com idade igual ou superior a 55 anos, na qual foram avaliados os fatores associados a quedas, foi observado que as maiores chances se encontravam em indivíduos do sexo feminino, com idade igual ou superior a 65 anos, qualidade de sono ruim, diagnóstico de depressão, com dificuldades de subir e descer escadas, considerados dependentes para atividades básicas e com baixa força de preensão palmar ${ }^{24}$. Todavia, neste estudo, não encontramos relação entre a força de preensão palmar e o risco de quedas nos idosos hospitalizados.

Avaliado o risco de queda por meio da Escala de Morse, percebe-se que a maioria dos participantes desta pesquisa apresenta risco de quedas moderado ou elevado. Em um estudo realizado com idosos hospitalizados, verificouse que mais de metade dos indivíduos apresentaram risco de queda de "médio" a "alto", quando associadas suas características, o diagnóstico e o tempo de hospitalização ${ }^{25}$. Na pesquisa de Ferrão et al. ${ }^{26}$, na qual foram avaliados 74 indivíduos institucionalizados, com média de idade de 83,69 anos, os autores perceberam que $44,3 \%$ destes idosos foram classificados como baixo risco de queda através da aplicação da Escala de Morse. Os autores supracitados apresentam resultados que corroboram os aqui apresentados.

Por intermédio da escala PS-ECOG, observa-se que os participantes poderiam realizar exercícios de natureza leve ou sedentária. Indo de encontro ao estudo de Pereira, Santos e Sarges ${ }^{13}$ que avaliaram a capacidade funcional de idosos oncológicos e observaram que a maioria pontuou em escores variando entre $2 \mathrm{e}$ 4, apresentando capacidade funcional alterada, nas quais os indivíduos eram incapazes de realizar atividades mesmo que de maneira leve.

Em um estudo realizado por Fhon et al. ${ }^{9}$ que teve por objetivo avaliar a relação entre as quedas e capacidade funcional em idosos, os autores identificaram que os indivíduos que sofreram quedas apresentaram incapacidade relacionada ao aumento da idade. Nossos dados corroboram este estudo, visto que em nossa amostra o maior impacto da capacidade funcional esteve relacionado ao aumento da idade e ao maior risco de quedas.

$\mathrm{O}$ risco de quedas encontrado em nosso estudo relacionou-se com o teste TUG aplicado à amostra, teste que tem sido utilizado com diversas finalidades, como, por exemplo, verificar a mobilidade. Em um estudo ${ }^{27}$, observou-se a relação entre o risco de quedas e o teste TUG, sendo que os indivíduos que caíram apresentaram TUG de $13,35 \pm 4,57$ segundos e os que não caíram 11,71 $\pm 3,61$ segundos $(p<0,001)$, valores semelhantes encontrados em nosso estudo.

A capacidade funcional prejudicada esteve relacionada ao aumento da idade em nosso estudo, o que vai ao encontro do estudo de Barbosa 
et al. ${ }^{28}$ no qual os autores identificaram que idosos com idade superior a 75 anos apresentaram maior incapacidade. Ainda, observamos que quanto menor a força muscular periférica, em todas as articulações mensuradas pela escala Kendall, maior o impacto na capacidade funcional dos idosos. Isso corrobora o estudo prévio no qual identificou-se uma associação da capacidade funcional com o desempenho muscular, ou seja, a força muscular em idosos ${ }^{29}$.

Destacamos, ainda, a importância do uso de protocolo de avaliação relacionados ao risco de quedas, à força muscular e à capacidade funcional em idosos hospitalizados e a necessidade de estudos futuros com essa população pautados nos respectivos aspectos.

\section{Conclusão}

A maioria dos idosos hospitalizados apresenta moderado ou elevado risco de quedas, força muscular periférica reduzida e maior impacto em sua capacidade funcional, embora sejam classificados como independentes. Ainda, o moderado ou elevado risco de quedas está relacionado ao maior impacto da capacidade funcional e ao maior tempo para realizar o teste TUG, enquanto o maior impacto da capacidade funcional, além de estar relacionado ao moderado ou ao elevado risco de quedas, está relacionado à baixa força muscular periférica.

\section{Referências}

1. Gasparotto L.P.R., Falsarella G.R, Coimbra A.M.V. As quedas no cenário da velhice: conceitos básicos e atualidades da pesquisa em saúde. Rev Bras Geriatr e Gerontol. 2014, v. 17, n. 1, p. 201-209.

2. Lopes GL, Santos MIP de O. Funcionalidade de idosos cadastrados em uma unidade da Estratégia Saúde da Família segundo categorias da Classificação Internacional de Funcionalidade. Rev Bras Geriatr e Gerontol. 2015, v. 18, n. 1: 71-83. doi: http://dx.doi.org/10.1590/1809-9823.2015.14013.
3. Pereira SRM, Buksman S, Perracini M, Py L, Barreto KML, Leite VMM. Sociedade Brasileira de Geriatria e Gerontologia. Projeto Diretrizes. Quedas em Idosos. São Paulo: Associação Médica Brasileira, Conselho Federal de Medicina; 2001. [acesso 10 nov. 2017]. Disponível: em: <http://www.portalmedico.org.br/ diretrizes/quedas_idosos.pdf $>$.

4. Piton D. A. Análise dos fatores de risco de quedas em idosos: estudo exploratório em instituição de longa permanência no município de Campinas. Dissertação (Programa de pós-graduação em Gerontologia). Campinas: Universidade Estadual de Campinas; 2004.

5. Nascimento, J.S.; Tavares, D. M. dos S. Prevalência e fatores associados a quedas em idosos. Texto Context. Enferm. 2016, v. 25: 2. doi: http://dx.doi. org/10.1590/0104-07072016000360015.

6. Veiga, B.; Pereira, R. A. B; Pereira, A. M. V. B.; Nickel, R. Evaluation of functionality and disability of older elderly outpatients using the Whodas 2.0. Revista Brasileira de Geriatria e Gerontologia, 2016, v. 19, n. 6): 1015-1021. doi: http://dx.doi.org/10.1590/198122562016019.150053.

7. Macedo, D. de O, Freitas, L. M. de; Scheicher M. E. Handgrip and functional mobility in elderly with different levels of physical activity. Fisioter $e$ Pesqui. 2014, v. 21, n. 2: 151-155. doi: http://dx.doi. org/10.1590/1809-2950/47321022014.

8. Carvalho, T. C.; Valle, A. P. do; Jacinto, A. F.; Mayoral, V. F. de S.; Boas, P. J. F. V. Impact of hospitalization on the functional capacity of the elderly: A cohort study. Revista Brasileira de Geriatria e Gerontologia, 2018, v. 21, n. 2: 134-142. doi: http:// dx.doi.org/10.1590/1981-22562018021.170143.

9. Fhon, J. R. S.; Coelho, S. C. F. W.; Vendruscolo, T. R. P.; Stackfleth, R.; Marques, S.; Rodrigues, R. A. P. Quedas em idosos e sua relação com a capacidade funcional. Revista Latino Americana de Enfermagem. 2012, v. 20, n. 5: 1-8.

10. Kendall, F. P.; Mccreary, E. K.; Provance, P. G. Músculos, provas e funções com postura e dor. 5. ed. São Paulo: Manole; 2007.

11. Bretan, O.; Elias Silva, J.; Ribeiro, O. R. Corrente JE. Risk of falling among elderly persons living in the community: assessment by the Timed up and go test. Braz J Otorhinolaryngol. 2013, v. 79, n. 1: 18-21. doi: http://dx.doi.org/10.5935/1808-8694.20130004. 
12. Urbanetto, J. de S.; Creutzberg, M.; Franz, F. et al. Morse Fall Scale: tradução e adaptação transcultural para a língua portuguesa. Rev da Esc Enferm da USP. 2013, v. 47, n. 3: 569-575. doi: http://dx.doi. org/10.1590/S0080-623420130000300007.

13. Pereira, E. E. B.; Santos, N. B. dos; Sarges, E. do S. N. F. Avaliação da capacidade funcional do paciente oncogeriátrico hospitalizado. Rev Pan-Amazônica Saúde. 2014, v. 5, n. 4: 37-44. doi: http://dx.doi. org/10.5123/S2176-62232014000400005.

14. Pereira, E. E. B.; de Souza, A. B. F.; Carneiro, S. R.; Sarges, E. do S. N. F. Funcionalidade global de idosos hospitalizados. Rev Bras Geriatr e Gerontol. 2014, v. 17, n. 1: $165-176$.

15. Rabelo, L. P. de O.; Vieira, M. A.; Caldeira, A. P.; Costa, S. de M. Perfil de idosos internados em um hospital universitário. Rev Min Enferm. 2010, v. 14, n. 3: 293-300. doi: http://dx.doi.org/S141527622010000300002 .

16. Maciel, M. G. Atividade física e funcionalidade do idoso. Motriz. 2010, v. 16, n. 4: 1024-1032. doi: http:// dx.doi.org/10.5016/1980-6574.2010v16n4p102.

17. Ferraro, N. S.; Cândido, A. da S. C. Percepção dos idosos acerca da atividade física na terceira idade. Id Line Rev Mult Psic. 2017, v. 11, n. 38: 597-611.

18. Maher, R. L.; Hanlon, J.; Hajjar, E. R. Clinical consequences of polypharmacy in elderly. Expert Opin Drug Saf. 2014, v. 13, n. 1: 57-65. doi: http:// dx.doi.org/10.1517/14740338.2013.827660.

19. Milos, V.; Bondesson, Å.; Magnusson, M.; Jakobsson, U.; Westerlund, T.; Midlöv, P. Fall risk-increasing drugs and falls: a cross-sectional study among elderly patients in primary care. BMC Geriatr. 2014, v. 14, n. 1: 40. doi: http://dx.doi.org/10.1186/1471-2318-14-40.

20. Van Strien, A. M.; Koek, H. L.; Van Marum, R. J.; Emmelot-Vonk, M. H. Psychotropic medications, including short acting benzodiazepines, strongly increase the frequency of falls in elderly. Maturitas. 2013, v. 74, n. 4: 357-362. doi: http://dx.doi. org/10.1016/j.maturitas.2013.01.004.

21. Nasrala Neto E.; Bittencourt, W. S.; Nasrala, M. L. S.; Oliveira, A. L. L. de; Souza, A. C. G. de; Nascimento, J. F. do. Correlations between low back pain and functional capacity among the elderly. Rev Bras Geriatr e Gerontol. 2016, v. 19, n. 6: 987-994. doi: http:// dx.doi.org/10.1590/1981-22562016019.150227.
22. Vieira, S. C. A. L.; Granja, K. S. B.; Exel, A. L.; Calles, C. A. N. A força muscular associada ao processo de envelhecimento. Cad Grad Ciências Biológicas e da Saúde. 2015, v. 3, n. 1: 93-102.

23. Cruz-Jentoft, A. J.; Baeyens, J. P.; Bauer, J. M. et al. Sarcopenia: European consensus on definition and diagnosis: Report of the European Working Group on Sarcopenia in Older People. Age Ageing. 2010, v. 39, n. 4: 412-423. doi: http://dx.doi.org/10.1093/ ageing/afq034.

24. Prato, S. C. F.; Andrade, S. M. de; Cabrera, M. A. S. et al. Frequency and factors associated with falls in adults aged 55 years or more. Rev Saude Publica. 2017; v. 51:1-11. doi: http://dx.doi.org/10.1590/s15188787.2017051005409.

25. Sardo, P. M. G.; Simões, C. S. O.; Alvarelhão, J. J. M.; Simões, J. F. F. L.; Melo, E. M. de; O. P. de. Fall risk assessment: retrospective analysis of Morse Fall Scale scores in Portuguese hospitalized adult patients. Appl Nurs Res. 2016, v. 31, n. 1: 34-40. doi: http://dx.doi.org/10.1016/j.apnr.2015.11.013.

26. Ferrão, S.; Henriques, A.; Fontes, R. Prevenção e controlo de quedas na Pessoa idosa institucionalizada em Lar - Avaliação de risco sistematizada através da aplicação da Escala de Avaliação de Risco de Queda de Morse, Teste Get Up and Go e Timed Get Up and Go. J Aging Inov. 2011, v. 1, n. 1: 14-22.

27. Aveiro, M. C.; Driusso, P.; Barham, E. J.; Pavarini, S. C. I.; Oishi, J. Mobilidade e risco de quedas de população idosa da comunidade de São Carlos. Cien Saude Colet. 2012, v. 17, n. 9: 2481-2488. doi: http:// dx.doi.org/10.1590/S1413-81232012000900028.

28. Barbosa, B. R.; Almeida, J. M. de; Barbosa, M. R.; Rossi-Barbosa, L. A. R. Avaliação da capacidade funcional dos idosos e fatores associados à incapacidade. Cien Saude Colet. 2014, v. 19, n. 8: 3317-3325. doi: http://dx.doi.org/10.1590/141381232014198.06322013.

29. Garcia, P. A.; Dias, J. M. D.; Rocha, A. S. D. S.; Almeida, N. C. de; Macedo, O. G. de; Dias, R. C. Relação da capacidade funcional, força e massa muscular de idosas com osteopenia e osteoporose. Fisioter Pesqui. 2015, v. 22, n. 2: 126-132. 